\title{
Moving Object Detection Using the Genetic Algorithm for Real Times Transportation
}

\author{
R. Aruna Jyothi, K. Ramesh Babu, Srinivas Bachu
}

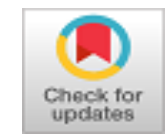

\begin{abstract}
Video surveillance, most commonly referred to as closed-circuit $T V$, is an industry over 30 years old and with a share of modifications in technology. To meet the requirements include: Better image quality, Reduction in costs, Size and scalability etc., video surveillance has experienced a number of technology shifts. For real-time traffic monitoring apps, we implement a process for the identification of objects. The suggested technique is a mixture of a GDSM, an enhanced version of the dynamic saliency map (DSM) and background subtraction. The experimental findings demonstrate the effective detection of moving objects by the suggested technique. Recent advances in vision technologies like distributed intelligent cameras have motivated scientists to create sophisticated apps for computer vision appropriate for embedded platforms. Simple and effective computer vision algorithms are needed in the integrated monitoring system with limited memory and computing resources.
\end{abstract}

Index Terms: Traffic surveillance, Genetic algorithm, Dynamic saliency map.

\section{INTRODUCTION}

The key in the application fields is moving object discovery in picture sequences [11, 12], for example, Visual robotic tracking, relationship between a human and a PC, video content compression and automatic traffic tracking. Particularly, vehicle discovery with stationary camera is a critical issue in movement administration, which is basic for the estimation of activity parameters, for example, vehicle check, speed, and stream [5-8]. As of late, foundation displaying is a normally utilized strategy to recognize moving items with settled camera. Be that as it may, exact discovery could be troublesome because of the potential changeability, for example, shadows thrown by moving items, non stationary foundation forms, and cover. Far reaching displaying of spatiotemporal data inside the video grouping is a key issue to powerfully section moving articles in the scene. Transient data is principal to deal with non stationary foundation forms. Direct procedures or likelihood circulations can be utilized to portray foundation changes

Revised Manuscript Received on October 30, 2019.

* Correspondence Author

R. Aruna Jyothi*, Department of ECE, Sai Tirumala NVR Engineering College, \& Marri Laxman Reddy Institute of Technology and Management, Dundigal, Hyderabad, India.

K. Ramesh Babu, Department of ECE, Sai Tirumala NVR Engineering College, Jonnalagadda, Gunutur, Andhra Pradesh, India.

Srinivas Bachu, Department of ECE, Marri Laxman Reddy Institute of Technology and Management, Dundigal, Hyderabad, India.

(c) The Authors. Published by Blue Eyes Intelligence Engineering and Sciences Publication (BEIESP). This is an open access article under the CC BY-NC-ND license (http://creativecommons.org/licenses/by-nc-nd/4.0/) from late perceptions $[9,10]$. In, the current history of pixel power is displayed by a blend of Gaussians, and the Gaussian blend is adaptively refreshed for each site to manage progression in foundation forms. Shading co event between continuous casings has likewise been proposed to show dynamic components of non stationary items. On the other hand, spatial data is imperative to comprehend the structure of the scene [1]. Gradient or edge highlights help enhance the unwavering quality of question discovery. In, spatial [13] co-event of picture varieties at neighboring squares is utilized to improve the discovery affectability. Spatial shading dispersion has additionally been proposed for frontal area question discovery. The organization of the paper as follows: The related works of research are discussed in chapter II. In chapter III describes the problems related to the video surveillance. Chapter IV deals with the methodology of real-time video surveillance using genetic algorithm. Results and discussions are explained in step by step manner in the chapter V. Finally, the summary and conclusions were incorporated in chapter VI.

\section{RELATED WORKS}

Y. Wang et al. [1] introduced an approach of moving vehicle location and cast shadow evacuation for video based activity checking. In view of contingent irregular field, spatial and transient conditions in activity scenes are detailed under a probabilistic discriminative system, where relevant requirements amid the recognition procedure can be adaptively balanced as far as information subordinate neighborhood association. Computationally effective calculation has been created to segregate moving cast shadows and handle non stationary foundation forms for ongoing vehicle recognition in video streams. Exploratory outcomes demonstrate that the proposed approach viably wires relevant conditions and powerfully identifies moving vehicles under substantial shadows even in gray scale video.

K. A. Joshi and D. G. Thakore et al. [2] presents an overview of different methods identified with video reconnaissance framework enhancing the security. The objective of this paper is to survey of different moving article identification and question following strategies. This paper concentrates on discovery of moving items in video observation framework at that point following the identified protests in the scene. Moving Item identification is first low level critical assignment for any video observation application. Recognition of moving article is a testing undertaking. Following is required in larger amount applications that require the

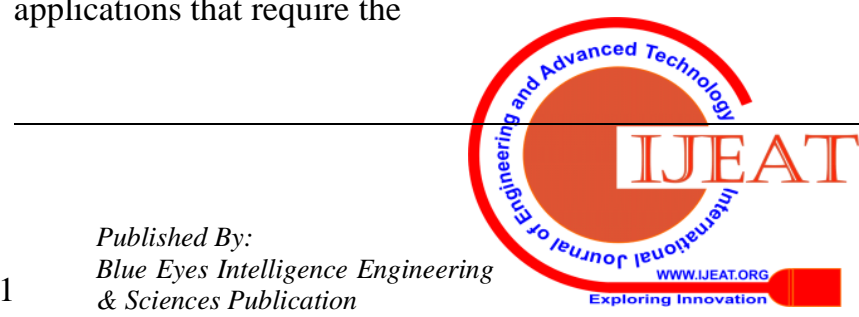


area and state of question in each edge. In this review, I portrayed Foundation subtraction with alpha, factual technique, Eigen foundation Subtraction and Worldly edge differencing to recognize moving item.

B. Rinner and W. Wolf et al. [3] introduced a circulated shrewd cameras (DSCs) are continuous conveyed installed frameworks that perform PC vision utilizing different cameras. This new approach has risen on account of a juncture of synchronous advances in four key orders: PC vision, picture sensors, inserted registering, and sensor systems. Preparing pictures in a system of appropriated savvy cameras presents a few complexities. Notwithstanding, we trust that the issues DSCs fathom are significantly more critical than the difficulties of planning and building a disseminated video framework. We contend that dispersed shrewd cameras speak to key segments for future inserted PC vision frameworks and that keen cameras will turn into an empowering innovation for some new applications. We compress shrewd camera innovation and applications, talk about momentum inclines, and recognize essential research challenges. C. Grana, and et al. [4] have discussed on moving visual items. Many ways to deal with moving item discovery for activity checking and video reconnaissance proposed in the writing depend on foundation concealment strategies. The most effective method to accurately and productively refresh the foundation model and how shadows are managed two of more identify and test elements of such procedures. This work shows a broadly useful approach for division of moving visual items (MVOs) in light of a protest level characterization in MVOs, apparitions and shadows. Foundation concealment needs a foundation model to be evaluated and refreshed: we utilize movement and shadow data to specifically avoid from the foundation show MVOs and their shadows, while holding phantoms. The shading data (in the HSV shading space) is misused to shadow concealment and, therefore, to improve both MVOs division and foundation refresh.

\section{PROBLEM IDENTIFICATION}

There were some problems which have been identified from the previous works while researching about the topic. Those are:

i. Foreground object detection

ii. Vehicle Classification

iii. Low Accuracy

The moving sensing of the genetic algorithm for traffic surveillance is the solution for the above difficulties. This method was discussed in section IV

\section{PROPOSED METHODOLOGY}

Moving article identification concentrates moving objects of intrigue, for example, cars, bikes and walkers in statically or dynamically based video groupings. Continuously traffic observation frameworks, moving item identification dependent on fixed CCTV cameras pictures, includes many testing issues are:

1. A number of moving items suddenly

2. Size variety, inadequately finished items

3. Alter in light conditions

4. Darkness and various impediments.
Distinguishing moving items has been generally connected in PC vision, so it pulls in serious consideration from analysts in the zone of picture handling. Notwithstanding, on the grounds that the surroundings in reality recordings are regularly very enunciated or even non-unbending, moving article recognition is as yet a difficult issue that should be additionally tended to. As showed there are for the most part three factors that make it progressively hard to distinguish moving articles from recordings.

1. The nearness of complex foundation, e.g., dynamic foundation with influencing trees,

2. Camera movement, e.g., tripod vibration,

3. Requiring earlier learning, e.g., preparing information for displaying the foundation.

Besides, most existing moving article recognition calculations are not smart or powerful enough for that they need client communication or experiential parameter tuning. For the most part, movement recognition techniques can be ordered into three approaches, specifically fleeting based, spatial-based, and joined methodology. For moving article recognition, movement signals are the most dependable data, so a mass of moving item location techniques are planned dependent on fleeting data, for example, outline contrast and foundation subtraction. Casing distinction is basic and can quick concentrates moving articles, however it is hard to get the whole form of the moving targets and effectively influenced by the annoyances of the foundation. Foundation subtraction, which expels the foundation model from the information pictures, is a typical way to deal with recognizes moving articles and has been generally utilized in pragmatic applications. Over the ongoing past, a large number of calculations for foundation demonstrating have been grown, for example, GMM (Gaussian Mixture Model), MRF (Markov Random Field) and BBM (Bayesian foundation model). Be that as it may, foundation displaying strategies require broad computational time to assess the foundation, and it is delicate to enlightenment changes. Furthermore, so as to show the foundation, the earlier learning, for example, the preparation information is required. The spatial-based item location is essentially connected in the space of article identification in static pictures and the outcomes are generally unwanted as the absence of fleeting data.

The human visual framework (HVS) has a noteworthy capacity to comprehend a scene and spotlight on the most fascinating (striking) objects. Recording the eye movement of eyewitnesses with eye-trackers has given some understanding into why individuals take a gander at specific territories in a picture. Continuous study is being done in developing computational visual output models to mirror the HVS's conduct. Some visual saliency models are roused by

psychological discoveries, some are computational, and others a blend of both. A great survey of saliency models is given. Abnormal state data, for example, sky, countenances, and people has been utilized as pointers to recognize outwardly notable locales. Anyway such explicit abnormal state data is prohibitive and not accessible in all pictures. 
Accordingly many bases up strategies utilize low level highlights for striking district identification. The general methodology is to utilize shading, surface, and recurrence segments to acquire a saliency map that features remarkable areas and smother uninteresting districts. Saliency maps have been utilized for a wide scope of uses in particular item recognition, picture and video rundown, video reconnaissance, enrollment and division of medicinal pictures, object following, coordinating people on foot from disjoint camera perspectives, and picture retargeting. A large number of the saliency strategies don't stifle false positives that happen because of exceptionally finished locales in pictures. In this article we present an algorithm for object detection that is appropriate for integrated monitoring. The technique suggested is a mixture of the GDSM and Background Subtraction. GDSM is Dynamics based Salt Maps (DSM)[3], needs less calculation, is more precise to detect objects and is richer in terms of noise and environmental changes. The GDSM's efficiency over standard DSM is based on the optimization of weights with a genetic algorithm while the initial DSM uses standardized weighs to create a saliency map.

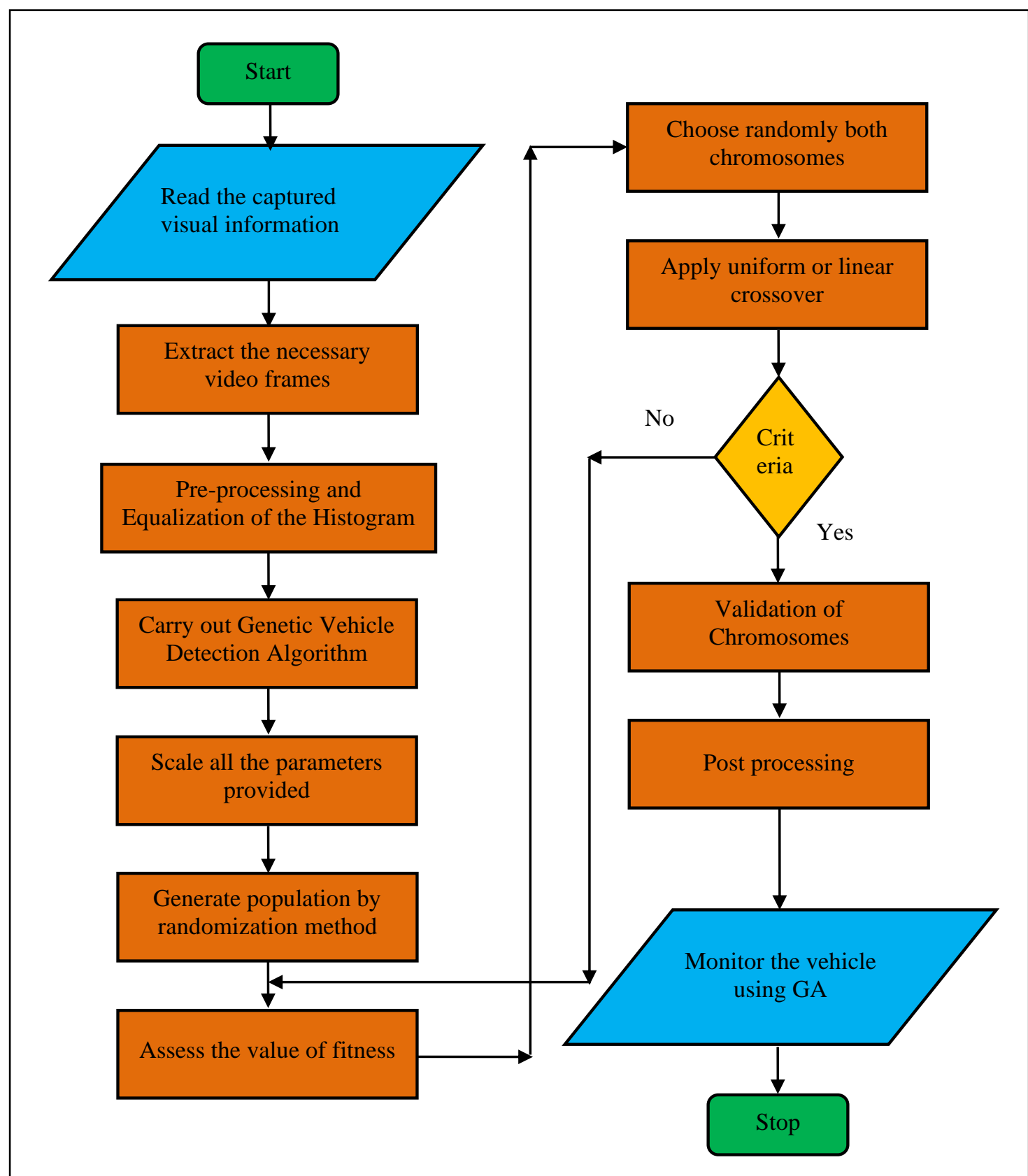

Figure 1: Flowchart of the Proposed Methodology [5, 12]

However, when you stop unpredictable in the center of the highway, GDSM fails to detect items. Thus, GDSM and Background Subtract (BS) are combined to detect moving items more accurately. BS helps detect tighter boundaries of objects and areas around them. We use a Gaussian average, which is known to be quick and easy, among multiple BS techniques. We suggest firstly, that an object be removed when a death event is detected and an object is added when a birth event is identified, to decrease the calculation cost while promoting a variable amount of motioning objects interacting. Figure 1 shows the methodology for real-time traffic monitoring of moving objects based on genetic algorithms. Check out the captured video. Download video in .mp4 format from the device. 
The feature encourages the .mp4 format we use strictly. Extract the necessary video frames. Many frames are present in the video because, set of frames is the video itself, so each frame is removed in this step. The Gaussian approach has concluded the extraction operation. Preparation of the image separated by the Gaussian technology. Pre-processing involves picture thinning, canny edge detection and gray scale conversion. First of all, the picture becomes a gray picture scale. The edges of the frames are marked with dotted lines using the Canny Edge detection method. The picture is now being diluted. The method is to draw a picture contour and compare it with the prior primary picture. If the picture borders match, this is shown, the image does not move, while if the boards do not match, then the image shows a certain movement. Histogram equalization. Drawing the histogram by a threshold value of 0.5 . The object's region is always thicker and it is common to compare the rest of the background to the denser region.Genetic vehicle detection algorithm performs to solving issues of Optimisation based on the natural method of choice that are both restricted and unconstrained. In order to achieve the highest outcome, the algorithm changes constantly a population of single alternatives. It produces alternatives to issues of optimization with naturally changing methods such as legacy, mutation, selection and crossover.Instate the parameters of hereditary calculation. Parameter scaling incorporates the way toward characterizing the qualities i.e., what number of chromosomes ought to be mulled over, how frequently it have to be outlined, and the quantity of qualities which are to be taken. Instate the chromosome age by randomization strategy. The populace is created as characterized already in the calculation. The best edge arrangement is obtained in the post-processing. Track the car with hereditary calculation help. The vehicle, which appears in the shaded green box, was accompanied by genetic algorithms.

\section{Advantages:}

i.Calculation burden is incredibly decreased since we need not process every pixel.

ii. Progressively precise and stable highlights are acquired contrasted with a solitary pixel.

iii. It is Efficient and strength.

\section{RESULTS AND DISCUSSIONS}

After selection of video we should give the frame number from where the detection should start. In the figure 2 we can see that the detection has started.

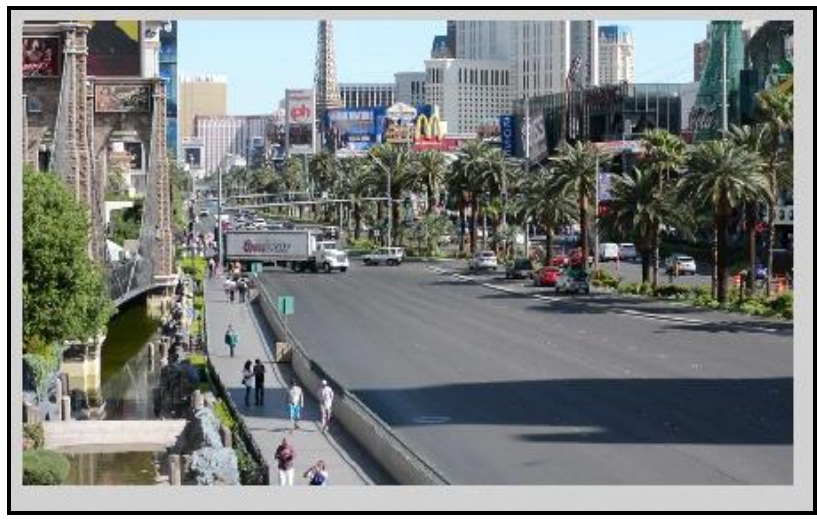

Figure 2: Detection Frame

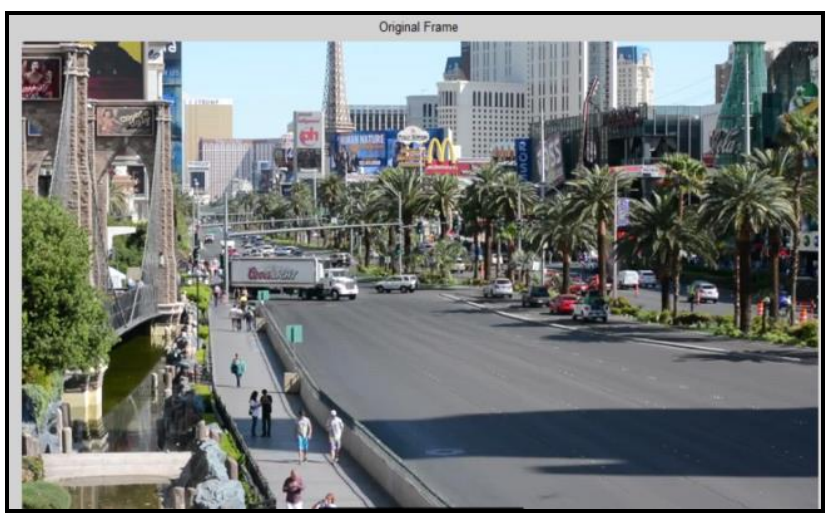

Figure 3: Original Frame

In figure 3 we can see that the original frame detection has started. After the original frame detection then frame separation process is done to separate all the frames i.e., shown in figure 4.

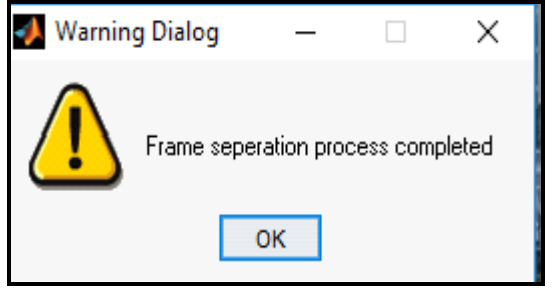

Figure 4: Frame Separation Process Window

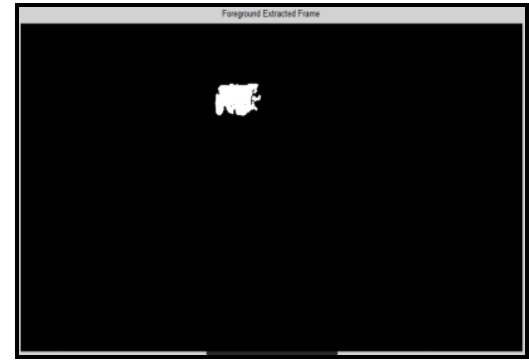

Figure 5: Foreground Extracted Frame

In figure 5 we can see that the foreground extracted frame. From the figure 4, it detects the small object that is moving.

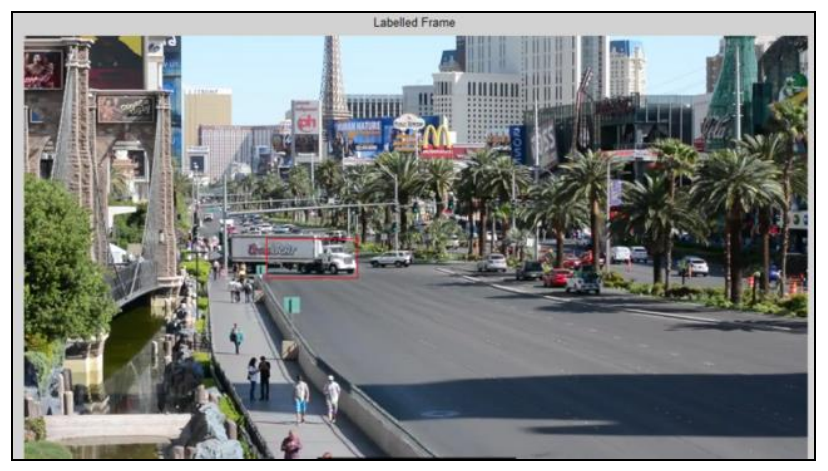

Figure 6: Label Frame

In figure 6 we can see the label frame on the particular object that is moving. In figure 7 we can see that the background detection process started detecting the constant objects and moving objects.

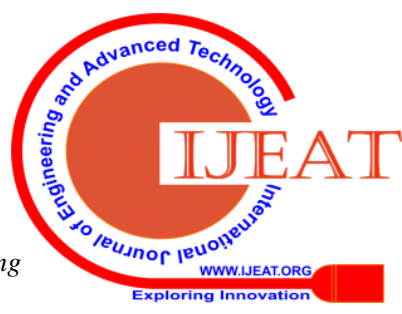




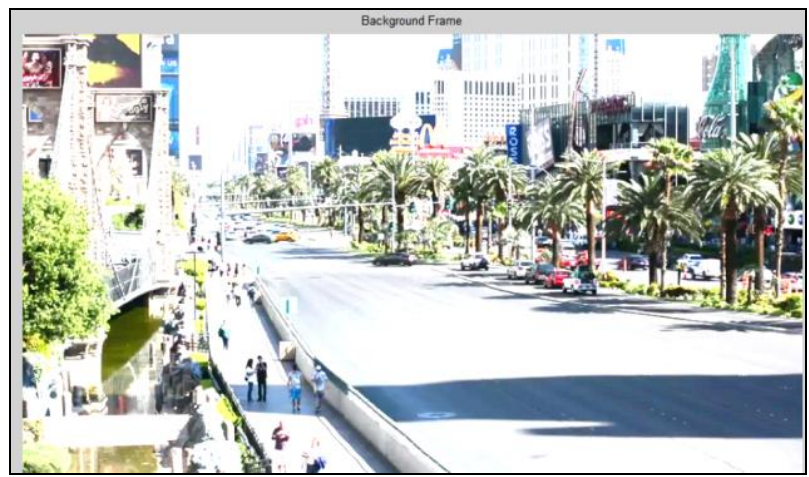

Figure 7: Background Detection

From figure 8 we see the selected frame that the objects are moving.
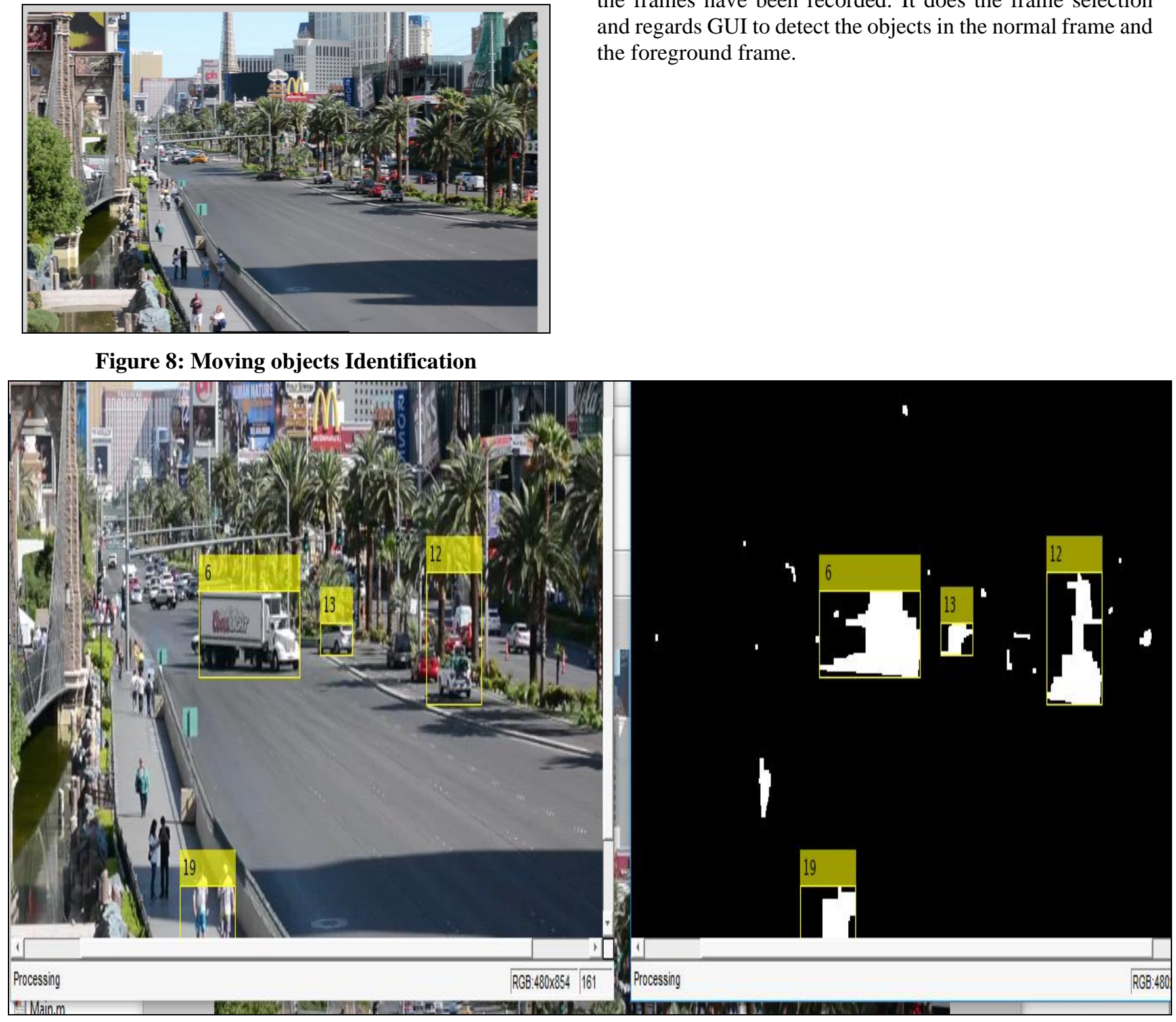

Figure 10: Moving Objects with Track ID's of Video

Figure 10 is the final output we can see the two frames displaying that detecting the moving objects. It detects the small object that is moving. This is generally used in the traffic surveillances to detect the speed of the object.

\section{Applications}

The human visual system (HVS) is able to comprehend a scene and concentrate its attention on the most interesting

Retrieval Number F8266088619/2019@BEIESP

DOI: 10.35940/ijeat.F8266.088619

Journal Website: www.ijeat.org

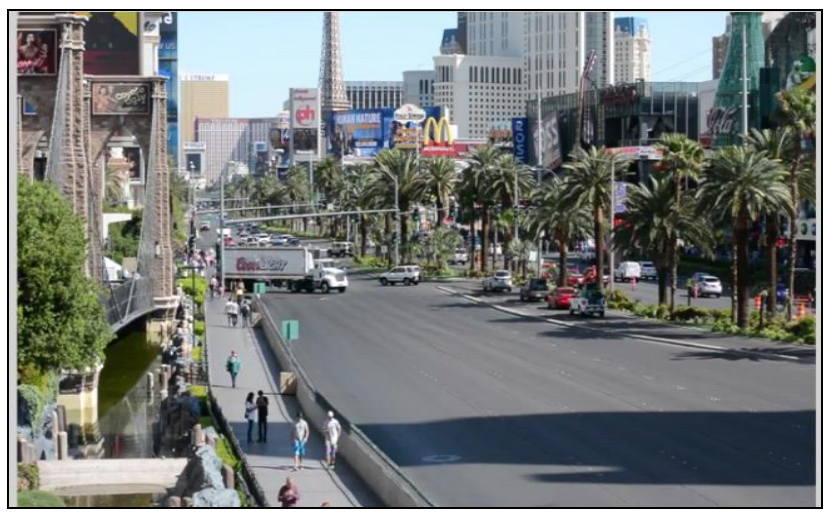

Figure 9: Final Frame of the Video Dataset

Figure 9 is the last frame before displaying the output that all the frames have been recorded. It does the frame selection and regards GUI to detect the objects in the normal frame and the foreground frame. objects. Recording eye movements of eye trackers observers has provided an insight into why individuals view certain regions in the picture.
Published By:

Blue Eyes Intelligence Engineering \& Sciences Publication

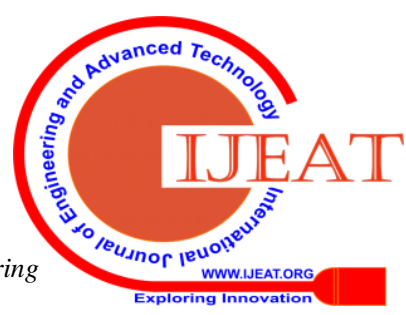


Current study is underway into creating visual saliency computational models to imitate the behaviour of the HVS [14]. Some visual models are influenced by cognitive results, some are computer-based and some combine. There is an outstanding examination of the salience models. Indicators for visually prominent areas are used as high-level data such as sky, faces and men.

- Detection of objects

- Summary of images and videos

- Video surveillance

- Medical image registration and segmentation

- Object monitoring

\section{CONCLUSION}

In this paper, we have suggested a better technique of object identification based on a map and background subtraction of genetic dynamics. The detection technique suggested for objects is quick, easy and efficient. It is therefore suited for integrated systems. The technique proposed is practical to effectively manage issues with shadow and occlusion. This technique is exceptionally valuable in recognizing an expansive number of vehicles, and it likewise diminished the preparing time, which was a noteworthy issue in the past works. In a further report, various components of a deformable 3-D vehicle display procured from the KVBs of the proposed strategy might be utilized to make strides characterization execution

\section{REFERENCES}

1. Y. Wang, "Real-time moving vehicle detection with cast shadow removal in video based on conditional random field," IEEE Trans. Circuits Syst. Video Technol., vol. 19, no. 3, pp. 437-441, 2009.

2. K. A. Joshi and D. G. Thakore, "A survey on moving object detection and tracking in video surveillance system," Int. J. Soft Comput. Eng. (IJSCE), vol. 2, no. 3, pp. 44-48, 2012.

3. J.-W. Woo, W. Lee, and M. Lee, "A traffic surveillance system using dynamic saliency map and svm boosting," Int. J. Contr. Auton. Syst., vol. 8, no. 5, pp. 948-956, 2010.

4. B. Rinner and W. Wolf, "An introduction to distributed smart cameras,” Proc. IEEE, vol. 96, no. 10, pp. 1565-1575, 2008.

5. M. Piccardi, "Background subtraction techniques: A review," in IEEE Int. Conf. Syst., Man and Cybern, 2004, vol. 4, pp. 3099-3104.

6. I. S. Kim, H. S. Choi, K. M. Yi, J. Y. Choi, and S. G. Kong, "Intelligent visual surveillance-A survey,” Int. J. Contr. Auton. Syst., vol. 8, no. 5, pp. 926-939, 2010.

7. R. Cucchiara, C. Grana, M. Piccardi, and A. Prati, "Detecting moving objects, ghosts, and shadows in video streams," IEEE Trans. Patt. Anal. Mach. Intell., vol. 25, no. 10, pp. 1337-1342, 2003.

8. P.-L. St-Charles, G.-A. Bilodeau, and R.Bergevin, "Flexible background subtraction with self-balanced local sensitivity," in IEEE Conf. Comput. Vision and Pattern Recogn. Workshop (CVPRW), 2014, pp. 414-419.

9. N. Paragios and R. Deriche, "Geodesic active contours and level sets for the detection and tracking of moving objects," IEEE Trans. Patt. Anal. Mach. Intell., vol. 22, no. 3, pp. 266-280, 2000.

10.D. E. Goldberg, Genetic Algorithms in Search, Optimization and Machine Learning. Reading, MA, USA: Addion-Wesley, 1989.

11.Bachu, Srinivas, and K. Manjunatha Chari, "A review on motion estimation in video compression," Signal Processing And Communication Engineering Systems (SPACES), 2015 International Conference on. IEEE, 2015.

12.Srinivas Bachu and K. Manjunathachari, "Adaptive Order Search and Tangent Weighted Trade off for H.264 in Motion Estimation,” Elsevier Journal: JKSU-Computer and Information Sciences, Vol. 30, Issue No. 2, pp. 249-258, April 2018.

13.Bachu, S. and Teja, N.R., "Fuzzy Holoentropy-Based Adaptive Inter-Prediction Mode Selection for H. 2ieo Coding," International Journal of Mobile Computing and Multimedia Communications (IJMCMC), Vol. 10, Issue No. 2, pp.42-60, April 2019.
14.Shijin Kumar P.S and Sudhan M.B., "A Hybrid Framework for Brain Tumor Detection and Classification using Neural Network," ARPN Journal of Engineering and Applied Sciences, Vol. 13, Issue 24, pp. 9631-9636, 2018. 